\title{
In situ functionalization of wet-spun fibre meshes for bone tissue engineering
}

\author{
Isabel B. Leonor*, Márcia T. Rodrigues, Manuela E. Gomes and Rui L. Reis \\ 3Bs Research Group - Biomaterials, Biodegradables and Biomimetics, Department of Polymer Engineering, University of Minho, \\ Headquarters of the European Institute of Excellence on Tissue Engineering and Regenerative Medicine, and Institute for Biotechnology \\ and Bioengineering (IBB), PT Government Associated Laboratory, Guimarães, Portugal
}

\begin{abstract}
Bone tissue engineering success strongly depends on our ability to develop new materials combining osteoconductive, osteoinductive and osteogenic properties. Recent studies suggest that biomaterials incorporating silanol $(\mathrm{Si}-\mathrm{OH})$ groups promote and maintain osteogenesis. The purpose of the present research work was to provide evidence that using wet-spinning technologies and a calcium silicate solution as a coagulation bath, it was possible to develop an in situ functionalization methodology to obtain 3D wet-spun fibre meshes with $\mathrm{Si}-\mathrm{OH}$ groups, through a simple, economic and reliable process. SPCL (blend of starch with polycaprolactone) fibre meshes were produced by wet-spinning, using a calcium silicate solution as a non-solvent and functionalized in situ with $\mathrm{Si}-\mathrm{OH}$ groups. In vitro tests, using goat bone marrow stromal cells (GBMSCs), showed that SPCL-Si scaffolds sustained cell viability and proliferation. Furthermore, high ALP activity and matrix production indicated that $\mathrm{Si}-\mathrm{OH}$ groups improve cellular functionality towards the osteoblastic phenotype. Using this methodology, and assembling several wet-spun fibre meshes, 3D meshes can be developed, aiming at designing osteoconductive/osteoinductive 3D structures capable of stimulating bone ingrowth in vivo. Copyright $\odot 2010$ John Wiley \& Sons, Ltd.
\end{abstract}

Received 12 August 2009; Accepted 25 February 2010

Keywords bone tissue engineering; marrow stromal cells; silanol groups; osteoconductive; biodegradable scaffolds; wet-spinning

\section{Introduction}

Biology plays an enormous influence on the demanding quest to develop biomedical technologies, as most modern strategies to design advanced materials are inspired in nature (Mitragotri and Lahann et al., 2009). Bone, one of the most remarkable materials in nature, is a dynamic and highly vascularized tissue in a continually remodelling process throughout the lifetime of an individual (Bonzani et al., 2006; Erik et al., 2009; Stevens et al., 2008). Its highly complex hierarchical structure has often been the basis for developing novel bone biomaterials. Tissue engineering of human bone is a

\footnotetext{
*Correspondence to: Isabel B. Leonor, 3Bs Research Group Biomaterials, Biodegradables and Biomimetics, Department of Polymer Engineering, University of Minho, Headquarters of the European Institute of Excellence on Tissue Engineering and Regenerative Medicine, AvePark, 4806-909 Taipas, Guimarães, Portugal.E-mail: belinha@dep.uminho.pt
}

complex process, as the functional development of bone cells requires that regulatory signals be temporally and spatially ordered (Kale et al., 2000). To mimic such a well-designed hierarchical structure, we must understand the morphological and physiological parameters of bone at a biological level to enhance our knowledge in designing novel approaches able to foster bone repair and regeneration.

Annually, more that 1000000 patients need a treatment for skeletal defects worldwide. Bone grafting is the second most frequent transplant procedure after blood transfusion, as evidenced by the enormous demand for therapies to enhance bone regeneration in different fields from plastic and reconstructive surgery to orthopaedic surgery and dental implantology (Drosse et al., 2008). Unfortunately, solutions available on the market are not completely satisfactory, as the requirements for scaffolding bone tissue engineering (TE) are complex. For a successful approach, bone implants should hold 


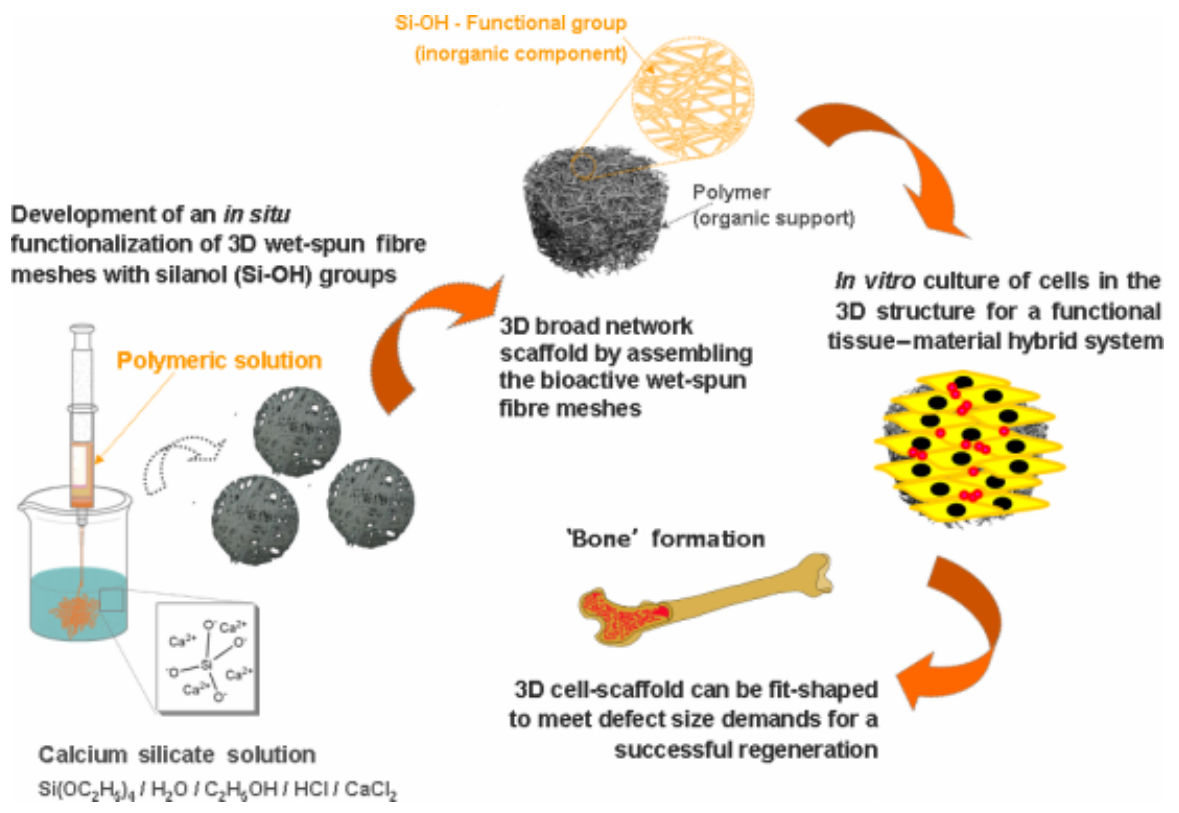

Figure 1. Schematic illustration of in situ functionalization of 3D wet-spun fibre meshes by the one-step approach, using the wet-spinning technique. These meshes are designed to successfully build stacking multilayer structures, resulting in a multifunctional 3D scaffold. These meshes can be designed into a stacking multilayer structure, resulting in a multifunctional 3D scaffold, combining both porosity and chemical cues for cell guidance towards bone formation/regeneration

osteoconductive and osteoinductive properties, together with osseointegration ability.

Up to now, one of the basic strategies of bone TE lays in using biocompatible and biodegradable scaffolds seeded with appropriate stem cells (Gurtner et al., 2007). Impressive advances have been achieved in the manufacture of biocompatible and biodegradable scaffolds, cell-seeding techniques and implantation protocols (Gurtner et al., 2007). So far, a diversity of polymeric porous scaffolds (naturally derived or synthetic matrices) have shown promising potential for bone TE applications (Kanczler and Oreffo et al., 2008). However, bone TE is currently compromised by its inability to produce load-bearing scaffolds, with limited osteoconductivity and osteoinductivity, and by the reduced biological cues to ensure the guidance of tissue regeneration compared to the current gold standard of allograft. On the other hand, pure calcium phosphate mineral-based scaffolds, which currently dominate the commercial bone-substitute materials market, lack the fibrillar protein component and are brittle, typically failing at compressive loads of $<5 \mathrm{MPa}$ (Andrew et al., 2009). As yet, the design criteria for an ideal scaffold for bone regeneration have not been fulfilled (Jones et al., 2009).

Thus, the challenge is the development of TE scaffolds by controlling the architecture, a well-interconnected pore network and overall surface chemistry through the incorporation of functional groups, to regulate cell behaviour and, simultaneously, to aid new tissue formation/regeneration. Aside from creating a stable and strong interface between the implant and bone tissue, adequate mechanical strength and degradation rate without undesirable by-products is also vital for the neobone to penetrate the defect to be restored/repaired.
Silicon ions play significant roles in bone and cartilage systems, acting on the physiological system most prominently during the growth and development of the skeletal system of higher organisms (Pietak et al., 2007). In addition, the release of Si and Ca ions has been reported to enhance bone regeneration by the upregulation and activation of genes in osteoprogenitor cells (Hench et al., 2009). Previous studies also showed that the presence of silanol $(\mathrm{Si}-\mathrm{OH})$ groups on polymeric materials is crucial to induce apatite formation in simulated body fluid (SBF) solution (Leonor et al., 2008; Oyane et al., 2003). The purpose of the present study is to provide evidence that, using wet-spinning technologies and a calcium silicate solution as a coagulation bath, it is possible to develop an in situ functionalization methodology to obtain 3D wetspun fibre meshes with $\mathrm{Si}-\mathrm{OH}$ groups through a simple, economic and reliable process, described in Figure 1.

With wet-spinning technologies it is possible to create hybrid materials with high levels of organization, with a particular array of chemical and physical properties, and also avoiding the thermal degradation of natural origin polymers compared to other techniques, such as the melt-spinning technique (Tuzlakoglu and Reis et al., 2009). To induce osteoconductive/osteoinductive behaviour, key points were considered to render a bioactive behaviour, as the classic ceramic materials, such as an organized arrangement of functional groups, silanol ( $\mathrm{Si}-\mathrm{OH})$ groups. Bioactive three-dimensional (3D) wet-spun fibre meshes were then fabricated via a one-step synthesis, in which the wet-spun polymer acts as a template mesh for incorporation of $\mathrm{Si}-\mathrm{OH}$ groups while it is precipitated in a calcium silicate solution (Figure 1). The in situ functionalization was performed under mild conditions that allowed the 
incorporation of biologically active molecules. Using this methodology, we were able to develop 3D wide-net mesh by assembling these thin wet-spun fibre meshes, which will be an important accomplishment for designing an osteoconductive/osteoinductive 3D structure capable of stimulating in vivo bone ingrowth. With this process, specific morphological features of the scaffolds can also be adjusted according to the location of implantation (e.g. thicker scaffolds for long bone or membrane-like scaffolds in maxillar applications).

\section{Materials and methods}

\subsection{Materials}

A biodegradable thermoplastic blend of corn starch with polycaprolactone (30:70 wt \%), designated as SPCL, was obtained from Novamont, Italy. Chloroform $\left(\mathrm{CHCl}_{3}\right)$ and methanol $\left(\mathrm{CH}_{3} \mathrm{OH}\right)$ were obtained from Sigma-Aldrich. Tetraethoxysilane (TEOS: $\left.\mathrm{Si}\left(\mathrm{OC}_{2} \mathrm{H}_{5}\right)_{4}\right)$ and calcium chloride $\left(\mathrm{CaCl}_{2}\right)$ were obtained from SigmaAldrich. Ethyl alcohol $\left(\mathrm{C}_{2} \mathrm{H}_{5} \mathrm{OH}\right)$ was obtained from Panreac.

\subsection{SPCL fibre mesh scaffold production}

In order to obtain a polymer solution with proper viscosity, SPCL was dissolved in chloroform at a concentration of $30 \% \mathrm{w} / \mathrm{v}$. The obtained polymer solution was loaded into a plastic syringe $(5 \mathrm{ml})$, with a metallic needle (internal diameter $0.8 \mathrm{~mm}$ ) attached to it. The syringe was connected to a programmable syringe pump (KD Scientific, World Precision Instruments, UK) to inject the polymer solution at controlled pumping rate to allow the formation of fibre mesh directly into the coagulation bath. The fibre mesh structure was formed during the processing by the random movement of the coagulation bath. Two different coagulation baths were used: (a) methanol as control; and (b) calcium silicate solution with a molar ratio, $\mathrm{Si}\left(\mathrm{OC}_{2} \mathrm{H}_{5}\right)_{4}: \mathrm{H}_{2} \mathrm{O}: \mathrm{C}_{2} \mathrm{H}_{5} \mathrm{OH}: \mathrm{HCl}: \mathrm{CaCl}_{2}$, of $1.0: 4.0: 4.0: 0.014: 0.20$ (Leonor et al., 2008; Oyane et al., 2003). Using methanol, the formed scaffolds were then dried at room temperature overnight in order to remove any remaining solvents, and were designated as SPCL. In the case of using calcium silicate solution, the fibre meshes were dried in air at $60^{\circ} \mathrm{C}$ for $24 \mathrm{~h}$, and designated as SPCL-Si. The scaffolds were cut into $5 \mathrm{~mm}$ diameter discs, followed by ethylene oxide sterilization before cellular studies.

\subsection{Assessing bone marrow cells behaviour seeded onto fibre mesh scaffolds}

Goat bone marrow stromal cells (GBMSCs) were isolated from the iliac crests of adult goats and expanded in basal medium composed of Dulbecco's modified Eagle's medium (DMEM; Sigma) supplemented with 10\% fetal bovine serum (FBS; Invitrogen) and 1\% antibiotic/antimicotic (A/B; Gibco). Cells were cryopreserved, expanded and subcultured twice [passage 2 (P2)] before being seeded onto the SPCL and SPCL-Si scaffolds at a concentration of $1.0 \times 10^{5}$ cells/scaffold. After the seeding, cell-scaffold constructs were maintained in basal medium for $24 \mathrm{~h}$ for cell attachment and then cultured in osteogenic medium, containing $\alpha$-modified Eagle's medium ( $\alpha$-MEM; Sigma), 10\% FBS, 1\% A/B and osteogenic supplements, ascorbic acid (50 $\mu \mathrm{g} / \mathrm{ml}$; Sigma), dexamethasone $\left(10^{-8}\right.$ M; Sigma) and $\beta$-glycerophosphate (10 mM; Sigma) for 7 and 14 days. Cell viability and proliferation were assessed by MTS quantification and dsDNA quantification assays, respectively, while cell morphology was assessed by scanning electron microscopy (SEM). Osteogenic differentiation was determined by the production of mineralized extracellular matrix, detected by Alizarin red staining (2\%; Sigma).

\subsection{Analyses of surface and respective solutions}

\subsubsection{Scanning electron microscopy}

SEM observation was performed with a Hitachi SU-70 UHR Schottky (Hitachi, Japan) attached with energydispersive electron X-ray spectroscopy (Bruker Quantax Esprit 1.8 EDS system, X-flash detector). Prior to any FE-SEM observations, all the scaffolds were sputtercoated with gold by ion sputtering and a graphite coating was used for EDS analysis. To observe the morphology of the GBMSCs cultured onto the fibre meshes, cell-scaffold constructs were washed in PBS, fixed in a $2.5 \%$ solution of gluteraldehyde (Sigma) overnight, dehydrated in a series of ethanol concentrations and air-dried before being sputter-coated.

\subsubsection{Micro-computed tomography $(\mu \mathrm{CT})$}

$\mu \mathrm{CT}$ evaluation of fibre meshes scaffolds was carried out using a high-resolution $\mu$ CT Skyscan 1072 scanner (Skyscan, Kontich, Belgium). X-ray scans were performed in triplicate, using a resolution of pixel size $5.86 \mu \mathrm{m}$ and integration time of $1.9 \mathrm{~s}$. The X-ray source was set at $40 \mathrm{keV}$ energy and $248 \mu \mathrm{A}$ current. Approximately 400 projections were acquired over a rotation range of $180^{\circ}$ with a rotation step of $0.45^{\circ}$. Datasets were reconstructed using standardized cone-beam reconstruction software (NRecon v1.4.3; SkyScan). Representative datasets of 200 slices were segmented into binary images with a dynamic threshold of 50-255 (grey values) to identify the organic and inorganic phase. These data was used for morphometric analysis (CTAnalyser, v 1.5.1.5, SkyScan) and to build 3D models (ANT 3D creator, v 2.4, SkyScan). The morphometric analysis included porosity, pore interconnectivity, mean pore size and fibre thickness. 


\subsubsection{Fourier transform attenuated total reflectance infrared spectroscopy (FTIR-ATR)}

FTIR-ATR was performed using IRPrestige-21 (Shimadzu, Japan) with an attenuated total reflectance (ATR). All spectra were recorded using at least 64 scans and $2 \mathrm{~cm}^{-1}$ resolution, in the spectral range $4000-600 \mathrm{~cm}^{-1}$.

\subsubsection{X-ray photoelectron spectroscopy}

XPS was performed on a VG ESCALAB 250iXL spectrometer (VG Scientific, UK). Pressure in the analysis chamber was around $8 \times 10^{-10} \mathrm{mBar}$. Due the non-conducting nature of the samples, it was necessary to use an electron flood gun to minimize surface charging. Neutralization of the surface charge was performed by using both a low-energy flood gun (electrons in the range $0-14 \mathrm{eV}$ ) and an electrically grounded stainless steel screen placed directly on the sample surface. The XPS measurements were carried out using monochromatic $\mathrm{Al}-\mathrm{K} \alpha$ radiation $(\mathrm{h} v=1486.92 \mathrm{eV}$ ) in a constant analyser energy mode (CAE) with a $100 \mathrm{eV}$ pass energy for survey spectra and $20 \mathrm{eV}$ pass energy for high-resolution spectra. Photoelectrons were collected from a take-off angle of $90^{\circ}$ relative to the sample surface. Charge referencing was adjusted by setting the lower binding energy C1s photopeak at $285.0 \mathrm{eV}$. Surface elemental composition was determined using the standard Scofield photoemission cross-sections. The identification of the chemical functional groups was obtained from the high-resolution peak analysis of C1s, and O1s envelopes by XPSPEAK 4.1 software.

\subsubsection{MTS assay}

MTS reagent, [3-(4,5-dimethylthiazol-2-yl)-5-(3-carboxymethoxyphenyl)-2-(4-sulfophenyl)-2-tetrazolium] was purchased from Promega. At each culturing time, cells were washed in PBS and then incubated in a MTS solution [one fraction of MTS reagent +5 fractions of basal culture medium without phenol red (Sigma)] for $3 \mathrm{~h}$ at $37^{\circ} \mathrm{C}$ in a $5 \% \mathrm{CO}_{2}$ environment. Afterwards, absorbance was read at $490 \mathrm{~nm}$.

\subsubsection{DNA quantification assay}

Proliferation of GBMSCs seeded onto the developed scaffolds was evaluated by double-stranded DNA quantification (dsDNA). For this purpose, a fluorimetric dsDNA quantification kit (PicoGreen, Molecular Probes) was used. The fluorescence was read using a microplate ELISA reader (BioTek) at an excitation of $485 / 20 \mathrm{~nm}$ and an emission of $528 / 20 \mathrm{~nm}$.

\subsubsection{Alizarin red staining}

Cell-scaffold constructs were removed from culture and fixed with $4 \%$ formalin (Sigma) solution overnight at $4^{\circ} \mathrm{C}$ and then washed and kept in PBS until usage. A photosensitive solution of $2 \%$ Alizarin red was prepared ( $\mathrm{pH}$ adjusted to $4.1-4.3$ ) to stain calcium ions that might be in the cell-scaffold constructs, resultant from mineral extracellular matrix produced by cells. Constructs were stained with Alizarin red solution for about 2 min, washed with PBS until removal of excess of stain, and allowed to dry. In the confocal picture, Alizarin red was counterstained with DAPI $(1 \mu \mathrm{g} / \mathrm{ml}$; Sigma) fluorescent stain. DAPI was prepared using a $0.1 \%$ solution of DAPI in PBS, stained for $15 \mathrm{~min}$ and then washed in PBS and protected from light until usage.

\section{Results and discussion}

Figure 2A, C show field-emission scanning electron microscopy (FE-SEM) micrographs and micro-computed tomography $(\mu \mathrm{CT})$ images of SPCL and SPCL fibre mesh scaffolds with $\mathrm{Si}-\mathrm{OH}$ groups (SPCL-Si), respectively. SPCL was considered to be a control of the wet-spinning technique. Furthermore, in this study, SPCL scaffolds also confirmed the chemical and biological importance of silanol groups in TE strategies.

FE-SEM micrographs revealed a typical SPCL fibre mesh structure with a random distribution of the fibres within the mesh and highly interconnected pores (Figure 2A). The porosity was about $85 \%$ and the fibre diameter was around $100 \mu \mathrm{m}$, as determined by $\mu \mathrm{CT}$ analysis. FE-SEM images in Figure 2C reveal a SPCL-Si fibre mesh structure with a large surface area and with highly interconnected pores. The porosity was around $56.84 \%$ (the pores are completely interconnected, as shown on the $\mu \mathrm{CT}$ image) and the fibre diameter was around $100 \mu \mathrm{m}$. At higher magnifications, the fibre surface of SPCL-Si (Figure 2D) seems to be smoother when compared with the control, SPCL (Figure 2B), which present a certain roughness. Such a difference is related to the processing and drying of the samples; in the case of SPCL, they are dried overnight until the methanol is completely evaporated, and for SPCL-Si they are dried in an oven at $60^{\circ} \mathrm{C}$ for $24 \mathrm{~h}$. Atomic force microscopy studies have been conducted on SPCL precipitated in methanol and in calcium silicate solution to measure the roughness of the fibres of the respective scaffolds.

The surface chemistry of SPCL and SPCL-Si was investigated by both Fourier transform infrared spectroscopy with attenuated total reflection (FTIR-ATR) and X-ray photoelectron spectroscopy (XPS). In the high wavenumber region, a very broad band in the range of $3550-3060 \mathrm{~cm}^{-1}$ with a higher intensity for SPCL-Si than for SPCL was observed, which can be assigned to the silanol $\mathrm{OH}$ hydrogen-bonded groups and to the silanol $\mathrm{OH}$ unassociated groups (Figure $2 \mathrm{E}$ ). In the low wavenumber region, the typical structural bands at 1727 and $1170 \mathrm{~cm}^{-1}$ were identified, which can be attributed to 

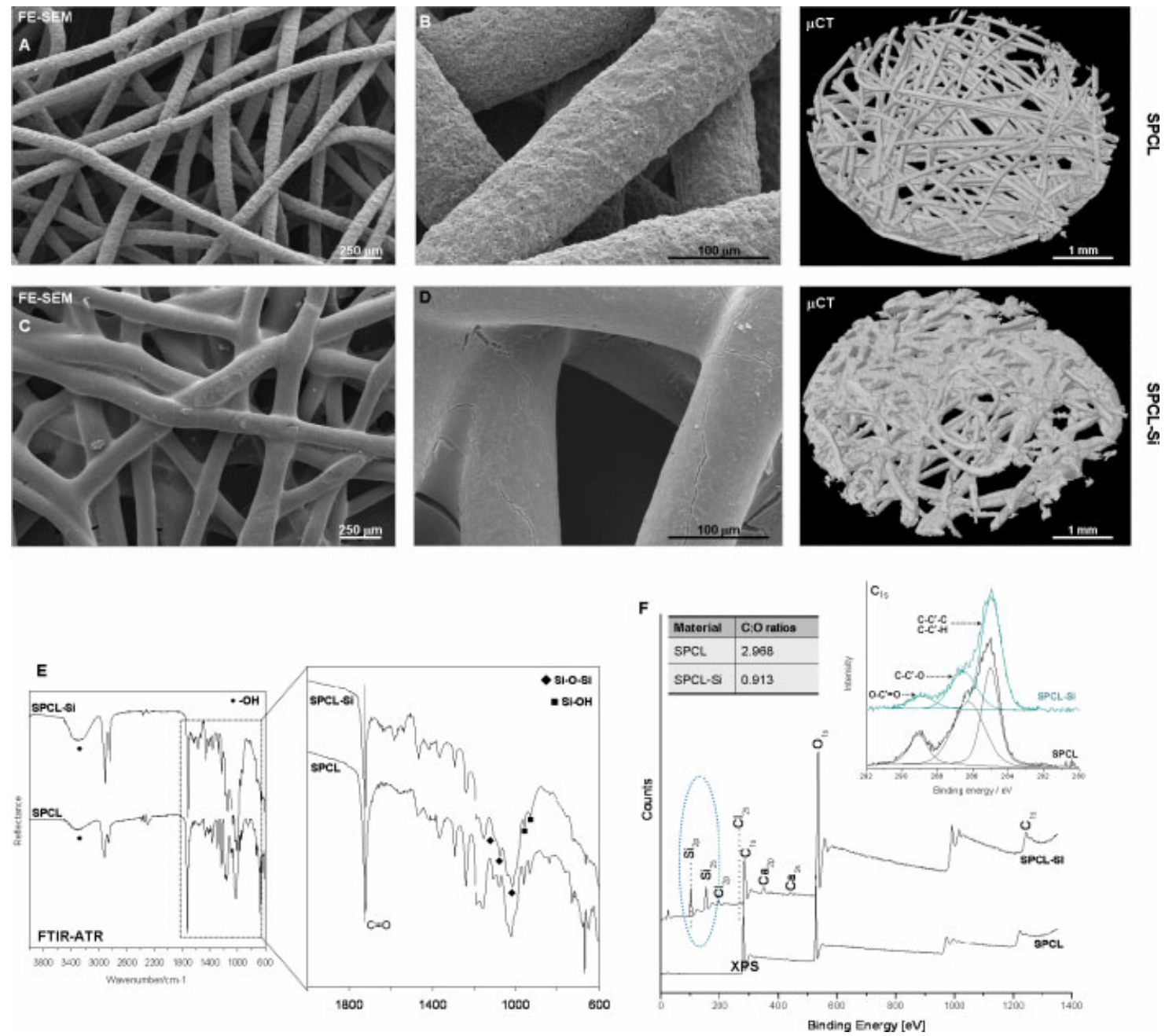

Figure 2. FE-SEM and $\mu$ CT images of SPCL (circular samples: diameter, $5 \mathrm{~mm}$; thickness, $1.0 \mathrm{~mm}$ ) (A) and SPCL-Si (circular samples, diameter, $5 \mathrm{~mm}$; thickness, $1.5 \mathrm{~mm}$ ) (C) fibre meshes produced by wet-spinning. Magnified FE-SEM images (B, D) of the structure presented in (A) and (C). (E) FTIR-ATR spectra of SPCL and SCPL-Si fibre meshes. (F) Large XPS spectra of the surface of SPCL and SPCL-Si fibre meshes. (Insets) Atomic C:O ratios and $\mathrm{C}_{1 \mathrm{~s}}$ XPS spectra of the surface of SPCL and SPCL-Si fibre meshes

the $\mathrm{C}=\mathrm{O}$ and $\mathrm{C}-\mathrm{O}$ absorption bands of PCL, respectively (Elzein et al., 2004; Kim et al., 2004; Pashkuleva et al., 2008). Using a calcium silicate solution as a coagulation bath, reflection peaks and a shoulder at $\sim 1080,1022$ and $1107 \mathrm{~cm}^{-1}$ are ascribed to siloxane ( $\mathrm{Si}-\mathrm{O}-\mathrm{Si}$ ) bonds and two bands $\sim 962$ and $\sim 948 \mathrm{~cm}^{-1}$ are assigned to $\mathrm{Si}-\mathrm{OH}$, typically observed in a silica gel (Bertoluzza et al., 1982). The presence of $\mathrm{Si}-\mathrm{OH}$ groups and $\mathrm{Si}-\mathrm{O}-\mathrm{Si}$ bonds suggests that TEOS was successfully hydrolysed to form $\mathrm{Si}-\mathrm{OH}$ groups and polycondensed to form a $\mathrm{Si}-\mathrm{O}-\mathrm{Si}$ network.

The results obtained from the XPS analysis are in good agreement with the FTIR data, confirming the incorporation of $\mathrm{Si}$ ions in the SPCL fibre mesh, using the calcium silicate solution as a coagulation bath, since these ions were not detected in the control samples (Figure 2F). The $\mathrm{C}_{1 \mathrm{~s}}$ core-level XPS spectra for SPCL fibre mesh scaffolds has three main peaks, one at $285 \mathrm{eV}$, corresponding to the main backbone carbon peak (polymeric $\mathrm{C}-\mathrm{C}$ or $\mathrm{CH}_{2}-\mathrm{CH}_{2}$ ), and another at about $286.4 \mathrm{eV}$ for-C-O-. The third peak at $288 \mathrm{eV}$ corresponds to the carbonyl/carboxyl bonded carbons [-C $\left.(=0) \mathrm{O}_{-}\right]$(Pashkuleva et al., 2008). In the case of SPCL-Si fibre mesh scaffolds, a decrease of the intensity of the carbon signal was clearly observed, specially for the bonds $(-\mathrm{C}-\mathrm{O}-)$ and $[-\mathrm{C}(=\mathrm{O}) \mathrm{O}-]$. This decrease could be related to the incorporation of silicon and oxygen and was confirmed by FT-IR measurements (see Figure 2E). Also, this hypothesis was confirmed by the presence of a new peak around $103.35-103.5 \mathrm{eV}$ in the $\mathrm{Si}_{2 \mathrm{p}}$ corelevel XPS spectra that is typically attributed to the $\mathrm{Si}-\mathrm{O}$ bond present in $\mathrm{SiO}_{2}$. As for the $\mathrm{O}_{1 \mathrm{~s}}$ region in SPCL-Si, two peaks were detected with binding energies of 532.26 and $531.68 \mathrm{eV}$, respectively. These signals correspond to the oxygen bound to the silicon, Si-O (Sprenger et al., 1990), and to possible water physisorbed on the surface. The signal at higher binding energy in the $\mathrm{Si}_{2 \mathrm{p}}$ core-level XPS spectra can be either oxide, $\mathrm{Si}-\mathrm{O}-\mathrm{Si}$, or hydroxide, $\mathrm{Si}-\mathrm{OH}$, since the position of the $\mathrm{Si}_{2 p}$ peak of the $\mathrm{Si}-\mathrm{O}$ bond is extremely close to that of the $\mathrm{Si}-\mathrm{OH}$ bond, and therefore it is difficult to confirm by $\mathrm{Si}_{2 \mathrm{p}}$. (Hayashi and Matsumoto et al., 1992; Sprenger et al., 1990). On the 


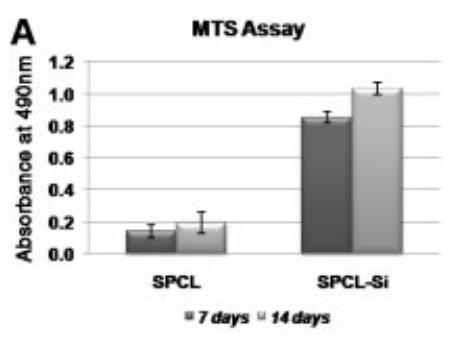

D

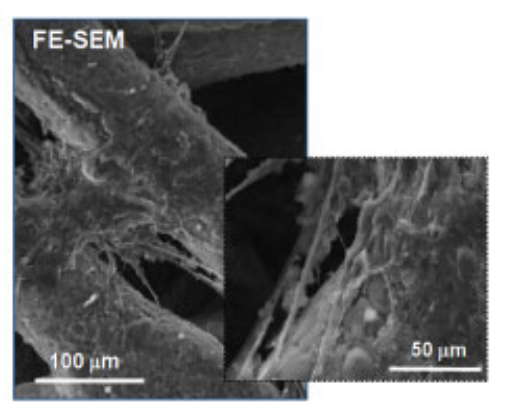

$\mathbf{F}$

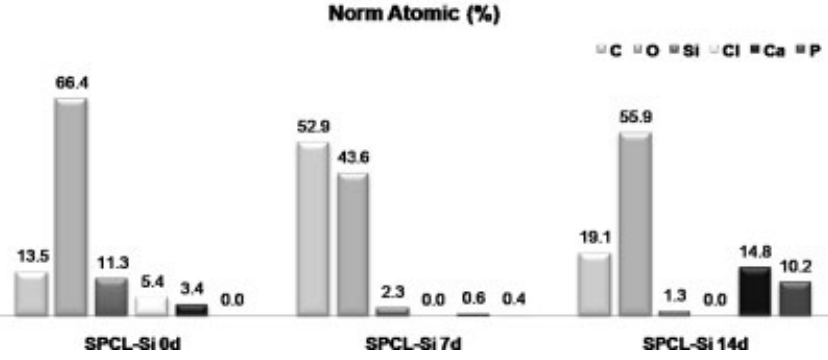

E
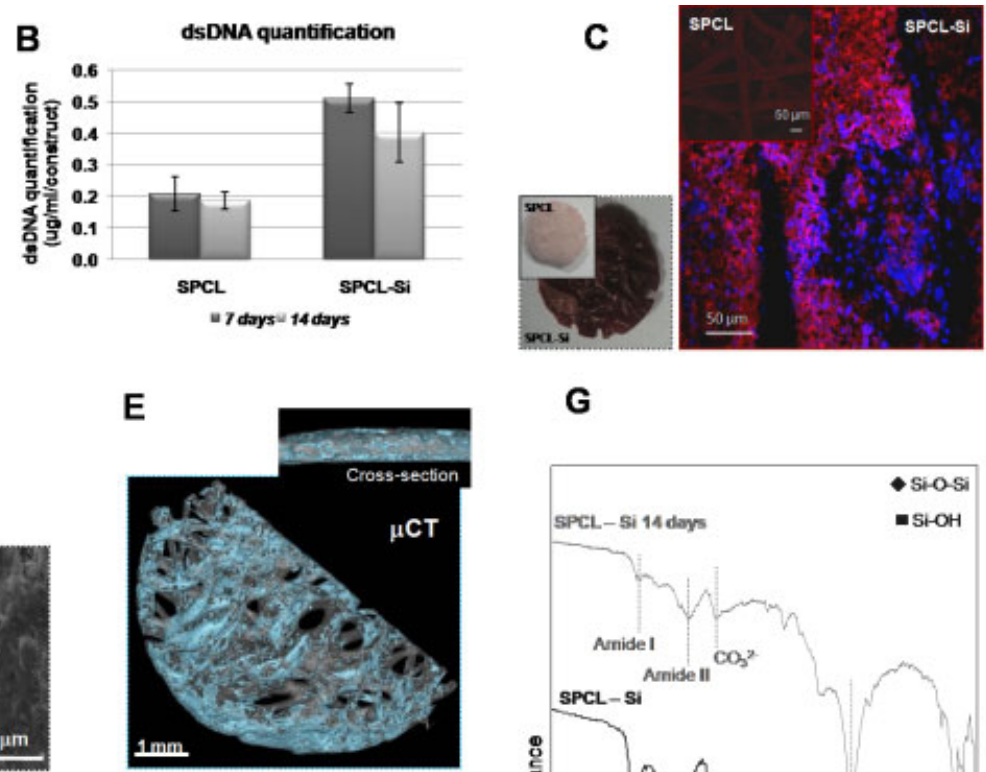

G

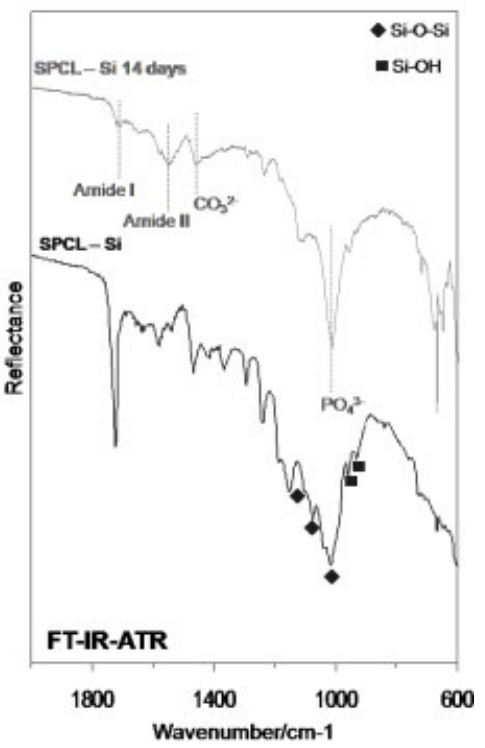

Figure 3. Biological assessment of scaffolds developed for bone TE. Cell viability was assessed using a MTS assay (A) and cell proliferation by DNA quantification (B) of GBMSCs seeded onto SPCL and SPCL-Si scaffolds after 7 and 14 days in osteogenic medium. Alizarin red staining (C), FE-SEM images (D), $\mu$ CT analysis (E) and FTIR-ATR (G) allowed evaluation of the formation of a calcified matrix in SPCL scaffolds with $\mathrm{Si}-\mathrm{OH}$ groups seeded with cells and cultured in osteogenic medium for 14 days. EDS analysis for calcium and phosphorus detection increment with culture time is also shown (F). In all assays, SPCL fibre meshes without $\mathrm{Si}-\mathrm{OH}$ groups were used as controls of the experiment. (Inset) Magnified FE-SEM image of the structure presented in (D)

other hand, the FTIR analysis allowed confirmation of the presence of $\mathrm{Si}-\mathrm{OH}$ groups on the surface of SPCL-Si (Figure 2E).

In terms of atomic concentration, a clear increase in the oxygen content and silicon incorporation on the surface of the SPCL-Si was observed, as compared to the control, SPCL. Also, the decrease of the C:O ratio in SPCL-Si scaffolds was more pronounced than in SPCL scaffolds, which again confirms the incorporation of $\mathrm{Si}-\mathrm{OH}$ group into the structure of SPCL (see table inset to Figure 2F) and is related with the decrease of the intensity of the carbon signal. According to the FTIR results, this increase in the oxygen is attributed to silanol $\mathrm{OH}$ hydrogen-bonded groups.

These results therefore suggested that only by precipitating a polymer solution, in this case SPCL, into the calcium silicate coagulation bath we are able to incorporate silanol ( $\mathrm{Si}-\mathrm{OH})$ groups into the surface of 3D wet-spun fibre mesh of SPCL. Note that using the method described herein, $\mathrm{Si}-\mathrm{OH}$ groups were successfully incorporated on the fibre surface during the fibre precipitation and, simultaneously, a bioactive 3D wet-spun fibre mesh was developed with a well-controlled porosity in one-step procedure. In this way, no further coating or either chemical modification of the fibre mesh is required to obtain a bioactive scaffold.

An in vitro biological assessment was performed to evaluate the osteoconductivity and osteogenic properties of novel wet-spun fibre mesh scaffolds of SPCL with or without (control) $\mathrm{Si}-\mathrm{OH}$ groups, using GBMSCs. The latter were seeded onto fibre mesh scaffolds for 7 or 14 days in osteogenic medium to determine their ability to support and sustain cell proliferation and osteogenic differentiation. Cell seeded/cultured fibre meshes were characterized by analysing cellular viability, proliferation and matrix mineralization as a result of osteogenic differentiation. Cell viability and proliferation are higher in cells seeded onto scaffolds with silanol groups, as observed in Figure 3A, B.

The maintenance in GBMSCs proliferation levels from 7 to 14 days in osteogenic culture medium (for both cells seeded on the control and $\mathrm{Si}-\mathrm{OH}$ scaffolds) is most probably associated to the GBMSCs osteogenic differentiation and consequent ECM mineralization, as evidenced by the positive Alizarin red staining in cell 
cultured SPCL-Si fibre mesh scaffolds after 14 days in osteogenic medium (Figure 3C).

FE-SEM micrographs revealed a good attachment of GBMSCs to the scaffolds, exhibiting a flat and elongated shape, typical of mature osteoblasts (Figure 3D). EDS analysis revealed the presence of calcium (Ca) and phosphorus $(\mathrm{P})$ in possible mineralization nodules, observed by FE-SEM. In Figure 3F, it can be observed that, after 7 days in osteogenic medium, there was an accentuated decrease in the $\mathrm{Ca}$ content that may be associated with the release of $\mathrm{Ca}$ ions (incorporated during the precipitation of SPCL into the calcium silicate solution) from the surface of the scaffold into the medium. This might be because starch is hydrophilic, and therefore allows the absorption of calcium ions from the calcium silicate solution, and hence the scaffolds released a fairly large amount of calcium ions into the osteogenic medium, where they were incubated in. As the incubation time increases in the osteogenic medium, the $\mathrm{Ca}: \mathrm{P}$ ratio also increases, likely because cells are producing mineralized ECM. The Ca: $\mathrm{P}$ atomic ratio determined in our scaffolds after 14 days in osteogenic culture was 1.45 (Figure 3F), slightly lower than that of hydroxyapatite present in natural bone, 1.67. In the case of silicon ions, their content on SPCL-Si surface decreases as a function of incubation time in osteogenic medium. This is related to the release of silicon ions from the SPCL-Si scaffold to the osteogenic medium, which results in the formation of $\mathrm{Si}-\mathrm{OH}$ groups, responsible for assisting the mineralization process of ECM (Figure 3F).

FTIR-ATR analysis of the samples cultured under the same conditions (Figure 3G) showed the presence of a well-defined band at $1018 \mathrm{~cm}^{-1}$ on SPCL-Si, which was attributed to phosphate groups $\left(\mathrm{PO}_{4}{ }^{3-}\right)$ and correlated to the production of mineralized ECM by the detection of reflectance peaks associated with the phosphate and carbonate groups. Also, amide bands at about $1620 \mathrm{~cm}^{-1}$ (amide I) and $1524 \mathrm{~cm}^{-1}$ (amide II) were detected, and therefore can be assigned to the protein matrix formed. Again, and due to density differences between SPCL polymer (either with or without functional groups) and calcified areas in the fibre meshes, mineralized ECM matrix was detected by $\mu \mathrm{CT}$ on both sides of SPCL-Si scaffolds seeded with GBMSCs after 14 days in osteogenic medium (Figure 3E). According to our results, ECM does not affect the porosity of the scaffold and it is homogeneously distributed, not only at the surface but throughout the scaffold, as shown. So, the results obtained show that silanol groups are important to stimulate the osteogenic differentiation of GBMSCs as well as the consequent production of calcified extracellular matrix, mimicking the regenerative process of native bone tissue.

\section{Conclusions}

We have demonstrated here that is feasible to produce, in a reliable and economical way, bioactive 3D fibre meshes, with $\mathrm{Si}-\mathrm{OH}$ functional groups on their surface, with a controlled pore size, shape and orientation, by using a simple one-step wet-spinning technique. Moreover, these fibre meshes sustained marrow stromal cells proliferation and differentiation into the osteogenic lineage. These data are more evident for cells seeded onto scaffolds with $\mathrm{Si}-\mathrm{OH}$ groups, indicating that $\mathrm{Si}-\mathrm{OH}$ groups improve cellular functionality towards the osteoblastic phenotype and, thus, providing further evidence of the enormous potential of these scaffolds for bone regeneration applications. Therefore, our aim is to offer a solution to the current clinical needs for a commercial alternative to allograft and autograft bone in several areas of the osteoregenerative field, by designing biomimetic hierarchical scaffolds using a one-step in situ functionalization wet-spinning technique. The promising biological outcome of SPCLSi wet-spun scaffolds is, thus, a platform for further investigation on these scaffolds for orthopaedics TE applications/strategies.

\section{Acknowledgements}

I. B. Leonor and M. T. Rodrigues thank the Portuguese Foundation for Science and Technology (FCT) for providing support: a post-doctoral scholarship (Grant No. SFRH/BPD/26648/2006) and a PhD scholarship (Grant No. SFRH/BD/30745/2006). This work was partially supported by the European NoE EXPERTISSUES (Grant No. NMP3-CT-2004500283).

\section{References}

Andrew MC, Nick JVS, Tom G, et al. 2009; Bone-like resorbable silk-based scaffolds for load-bearing osteoregenerative applications. Adv Mater 21: 75-78.

Bertoluzza A, Fagnano C, Morelli MA, et al. 1982; Raman and infrared spectra on silica-gel evolving toward glass. $J$ NonCryst Solids 48: 117-128.

Bonzani IC, George JH, Stevens MM. 2006; Novel materials for bone and cartilage regeneration. Curr Opin Chem Biol 10: 568-575.

Drosse I, Volkmer E, Capanna R, et al. 2008; Tissue engineering for bone defect healing: an update on a multi-component approach. Injury 39(suppl 2): S9-20.

Elzein T, Nasser-Eddine M, Delaite C, et al. 2004; FTIR study of polycaprolactone chain organization at interfaces. $J$ Colloid Interface Sci 273: 381-387.

Erik DS， Shawn GA， Samuel IS. 2009, Enzyme-directed templating of artificial bone mineral. Adv Mater 21 : 425-430.

Gurtner GC, Callaghan MJ, Longaker MT. 2007; Progress and potential for regenerative medicine. Annu Rev Med 58: 299-312.
Hayashi Y, Matsumoto K. 1992; Determination of surface silanol group on silicate-glasses using static Sims. Nippon Seramikkusu Kyokai Gakujutsu Ronbunshi [J Ceram Soc Japan] 100: 1038-1041.

Hench LL. 2009; Genetic design of bioactive glass. J Eur Ceram Soc 29: 1257-1265.

Jones JR. 2009; New trends in bioactive scaffolds: the importance of nanostructure. $J$ Eur Ceram Soc 29: 1275-1281.

Kale S, Biermann S, Edwards C, et al. 2000; Three-dimensional cellular development is essential for ex vivo formation of human bone. Nat Biotechnol 18: 954-958. 
Kanczler JM, Oreffo RO. 2008; Osteogenesis and angiogenesis: the potential for engineering bone. Eur Cell Mater 15: 100-114.

Kim HW, Knowles JC, Kim HE. 2004, Development of hydroxyapatite bone scaffold for controlled drug release via $\operatorname{poly}(\varepsilon$-caprolactone) and hydroxyapatite hybrid coatings. $J$ Biomed Mater Res $B$ Appl Biomater 70: 240-249.

Leonor IB, Baran ET, Kawashita M, et al. 2008; Growth of a bonelike apatite on chitosan microparticles after a calcium silicate treatment. Acta Biomater 4: 1349-1359.
Mitragotri S, Lahann J. 2009; Physical approaches to biomaterial design. Nat Mater 8: 15-23.

Oyane A, Kawashita M, Nakanishi K, et al. 2003; Bonelike apatite formation on ethylene-vinyl alcohol copolymer modified with silane coupling agent and calcium silicate solutions. Biomaterials 24 1729-1735.

Pashkuleva I, Azevedo HS, Reis RL. 2008; Surface structural investigation of starchbased biomaterials. Macromol Biosci 8: 210-219.

Pietak AM, Reid JW, Stott MJ, et al. 2007; Silicon substitution in the calcium phosphate bioceramics. Biomaterials 28: 4023-4032.

Sprenger D, Bach H, Meisel W, et al. 1990; Xps study of leached glass surfaces. $J$ Non-Crystal Solids 126: 111- 129 .

Stevens MM. 2008; Biomaterials for bone tissue engineering. Mater Today 11: 18-25.

Tuzlakoglu K, Reis RL. 2009; Biodegradable polymeric fiber structures in tissue engineering. Tissue Eng B Rev 15: $17-27$ 